\title{
Reintroduction of Imatinib in GIST
}

\section{T. Reid}

Published online: 7 August 2013

(C) Springer Science+Business Media New York 2013

\section{Introduction}

Imatinib is the only first-line targeted therapy approved to treat patients with gastrointestinal stromal tumors (GIST), both as adjuvant therapy following resection and in the advanced/ metastatic setting [1-3]. Imatinib has significantly improved outcomes for patients with metastatic GIST compared to historical controls treated with traditional chemotherapy, radiation therapy, and/or best supportive care (BSC) [4]. Disease control ranges from 70 to $85 \%$; median progression-free survival (PFS) is 29 months, and median overall survival (OS) is 57 months $[4,5]$. Unfortunately, only 3-5\% of patients with unresectable or metastatic GIST treated with imatinib experience complete tumor response (CR), and the 3-year PFS rate is $31 \%[5,6]$. More than $50 \%$ of patients who benefit from imatinib develop progressive disease (PD) within 2 years, which poses an important clinical problem [6].

Another clinical challenge comes from the fact that patients sometimes opt to stop imatinib therapy due to treatmentrelated adverse events (AEs) or other reasons but whether these interruptions favor or limit emergence of drug-resistant clones is unclear [7]. Moreover, optimal duration of adjuvant imatinib treatment for patients with localized resected disease remains undetermined and whether patients with recurrent disease may benefit from reintroduction of imatinib upon relapse later has not been extensively investigated.

Disease progression or recurrence after treatment with traditional cytotoxic anticancer chemotherapy often indicated that treatment failed (due to resistance) and could not be used again (e.g., in case of relapse) [8]. However, accumulating evidence suggests that in some patients, targeted therapy with tyrosine kinase inhibitors (TKI) can be effectively reintroduced after prior use to control tumor growth. For example, patients with chronic myeloid leukemia (CML) are typically treated with daily imatinib indefinitely, unless they experience relapse or unacceptable toxicity [9]. In the Stop Imatinib (STIM) trial,
T. Reid $(\bowtie)$

Department of Hematology/Oncology, Moores UCSD Cancer Center, University of California, 3855 Health Sciences Drive, La Jolla, San Diego, CA 92093, USA

e-mail: tonyreid@ucsd.edu 
Mahon et al. found that molecular relapse occurred in $54 \%$ of patients with CML who interrupted treatment (median follow-up, 17 months) [9]. When imatinib (400 mg/day) was reintroduced in those patients, all of them regained complete molecular response after a median of 3 months, regardless of initial duration of imatinib therapy. These results suggest that discontinuation does not lead to acquired, secondary resistance (Box 1) and that imatinib reintroduction is feasible in patients with CML [9]. Herein, we review the evidence supporting the reintroduction of imatinib in patients with GIST who were previously treated with imatinib in the adjuvant or advanced/ metastatic setting. The data indicate that interruption of imatinib does not preclude its subsequent use after GIST recurrence or progression.

Box 1: Primary versus secondary resistance

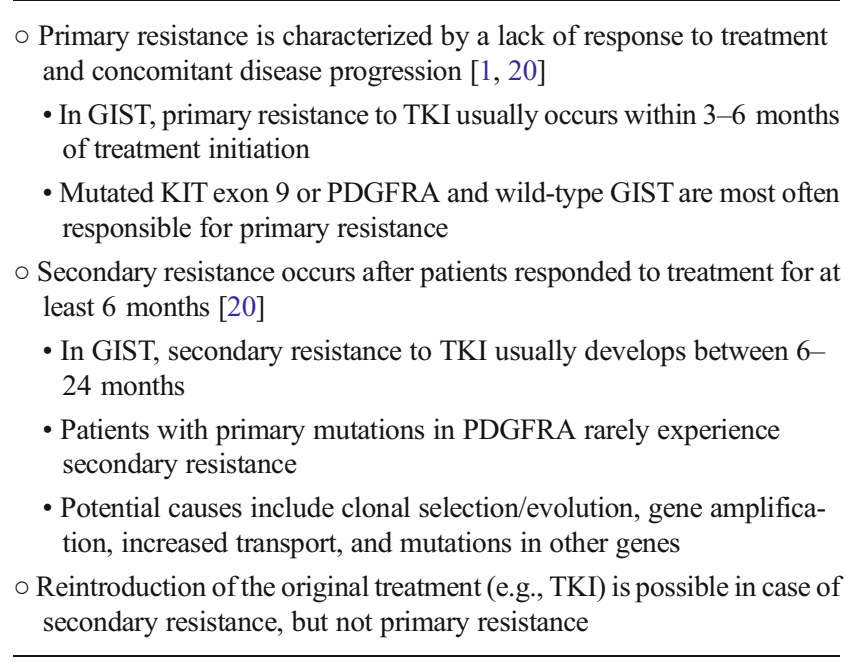

GIST, gastrointestinal stromal tumor; PDGFRA, plateletderived growth factor receptor alpha; TKI, tyrosine kinase inhibitors

Reintroduction of Imatinib in the Advanced/Metastatic Setting

For patients with advanced/metastatic GIST, current National Comprehensive Cancer Network (NCCN) guidelines recommend that imatinib therapy be continued indefinitely or until disease progression [1]. The NCCN guidelines also caution that discontinuing imatinib/TKI therapy may accelerate the pace of disease progression [1].

Because imatinib-related AEs are typically mild to moderate and manageable, chronic administration for GIST is generally feasible. However, even mild AEs may prompt patients to request a treatment interruption/holiday if experienced chronically [7]. The phase III BFR14 trial thus investigated whether imatinib may be stopped in patients whose disease is controlled [10]. Unfortunately, data indicated that for patients who achieved disease control for 1 year while on imatinib treatment, subsequent interruption of imatinib was associated with a major risk of disease progression: median time to progression (TTP) was 6 months and more than $70 \%$ of the patients had relapsed 1 year after discontinuing imatinib [10]. Importantly, $92 \%$ of the patients who reintroduced imatinib during GIST progression regained tumor control [10]. Similarly, the BFR14 study showed rapid GIST progression when patients stopped imatinib after 1,3 , or 5 years of treatment with disease control (Table 1) [7]. Results also showed that patients who restarted imatinib regained tumor control (Table 1), indicating that imatinib can be reintroduced upon disease progression. Interruption of imatinib does not prevent subsequent use to control GIST [7]. Indirectly, these results also suggest that imatinib reintroduction can be beneficial in patients who interrupt treatment of advanced/metastatic GIST due to AEs (or other personal reasons) but are not necessarily resistant to imatinib. However, the high relapse rate suggests that it is not advisable to discontinue imatinib therapy.

\section{Reintroduction of Imatinib after Completion of Adjuvant Therapy}

DeMatteo et al. [11] were the first to report rapid/early disease recurrence after discontinuation of imatinib in the adjuvant setting. In the phase III American College of Surgeons Oncology Group (ACOSOG) Z9001 trial, patients who had complete resection of primary GIST $\geq 3 \mathrm{~cm}$ were randomized to receive imatinib $400 \mathrm{mg} /$ day $(N=359)$ or placebo $(N=354)$. After a follow-up of 19.7 months, $20 \%$ of patients in the placebo group had either tumor recurrence or died versus $8 \%$ in the imatinib group. Imatinib was well tolerated and improved the 1-year recurrence-free survival rate (RFS, $98 \%$; $95 \%$ confidence interval (CI), 96-100) compared with placebo (RFS, $83 \%$; $95 \%$ CI, 78-88). The hazard ratio was 0.35 (95\% CI, 0.22-0.53; $p<0.0001)$. Notably, the rate of recurrence increased in the imatinib group after approximately 18 months (i.e., 6 months after completion of therapy), suggesting that it

Table 1 Reintroduction of imatinib can rescue progression of advanced/ metastatic GIST that occurs following treatment interruption

\begin{tabular}{llll}
\hline Years of imatinib before randomization & 1 year & 3 years & 5 years \\
\hline $\begin{array}{l}\text { Randomized patients }(N) \text { : } \\
\quad \text { interruption/continuation }\end{array}$ & $32 / 28$ & $25 / 25$ & $14 / 13$ \\
$\begin{array}{l}\text { Median PFS (months): } \\
\quad \text { interruption/continuation }\end{array}$ & $7 / 29$ & $9 / \mathrm{NR}$ & $13 / \mathrm{NR}$ \\
$\begin{array}{l}\text { Patients with tumor control } \\
\quad \text { after imatinib reintroduction (\%) }\end{array}$ & 92 & 100 & 86 \\
\begin{tabular}{l} 
Median follow-up (months) \\
\hline
\end{tabular} & 74 & 47 & 18 \\
\hline
\end{tabular}

Adapted from Blay et al. by the permission of Oxford University Press [7] GIST gastrointestinal stromal tumor, NR not reached, PFS progressionfree survival 
may not be advisable to interrupt imatinib treatment following GIST resection [11].

In the phase III Scandinavian Sarcoma Group of the Arbeitsgemeinschaft Internistische Onkologie (SSGXVIII/ AIO) trial, patients with a high risk of recurrence following GIST resection were randomly assigned to receive 12 months $(N=199)$ or 36 months $(N=198)$ of imatinib $400 \mathrm{mg}$ /day. At a median follow-up of 54 months, disease had recurred in $27.1 \%$ of patients in the 1-year group versus $13.6 \%$ of patients in the 3 -year group. These patients were then retreated with imatinib at $100 \mathrm{mg} /$ day $(N=3), 400 \mathrm{mg} /$ day $(N=71), 600 \mathrm{mg} /$ day $(N=1)$, or $800 \mathrm{mg} /$ day $(N=6)$ [12]. Clinical benefit was observed in $84.8 \%$ of patients and was not different between the 1- and 3-year arms $(p=0.385)$ : $32.6 \%$ had CR, $30.4 \%$ had partial response (PR), and $21.7 \%$ had stable disease (SD). Similarly, median TTP (35.7 months) was not different between the two arms $(p=0.289)$ [12]. These results suggest that more than $84 \%$ of the patients who completed adjuvant imatinib and received imatinib as first-line treatment for recurring GIST responded to imatinib reintroduction, independent of the length of prior imatinib therapy in the adjuvant setting ( 1 versus 3 years) [12, 13]. Moreover, the response rate did not differ markedly from that of imatinib-naive patients, regardless of the duration of prior adjuvant imatinib treatment $[12,13]$. Based on these data, NCCN guidelines recommend at least 1 year of imatinib treatment following resection of primary GIST and at least 3 years of therapy for patients at high risk of recurrence [1].

In the SSGXVIII/AIO trial, Joensuu et al. [13] also showed that only $2 \%(4 / 199)$ and $6 \%(12 / 198)$ of the patients developed GIST recurrence while receiving adjuvant imatinib for 12 and 36 months, respectively, compared with $40 \%$ of patients treated with surgery alone (historical controls with a median follow-up of 24 months) [14]. These results suggest that acquired resistance to adjuvant imatinib was infrequent, especially since both arms included patients with mutations known to be less sensitive or resistant to imatinib (e.g., platelet-derived growth factor receptor alpha [PDGFRA] D842V) [13].

In a case report, Kang [15] described a patient who was at high risk of recurrence (after undergoing complete resection of a primary GIST) and received 2 years of imatinib therapy. Ten months after completion of the adjuvant treatment, a computed tomographic (CT) scan revealed disease recurrence. The patient was re-treated with imatinib $400 \mathrm{mg} /$ day and, 1 month later, a PR was observed (by Response Evaluation Criteria In Solid Tumors [RECIST]) with a $43 \%$ decrease in tumor size and mild AEs (grade 1 edema, anemia, and fatigue). The PR was maintained for 33 months before the disease progressed again. The patient was then treated with a dose escalation to $800 \mathrm{mg} /$ day and was still being monitored at the time of publication.
Overall, these results suggest that sensitivity to imatinib is not compromised by prior exposure to adjuvant imatinib and that imatinib reintroduction remains an option for patients who experience GIST recurrence after completing the recommended adjuvant treatment (Table 2).

\section{Reintroduction of Imatinib after Neoadjuvant Therapy}

Although imatinib is not approved by the US Food and Drug Administration for this indication, the NCCN mentions that imatinib may be used as neoadjuvant prior to surgery to downstage a tumor and improve the probability of R0 resection and/or reduce morbidity (Fig. 1) [1]. Many investigations have shown the benefits of perioperative imatinib. In a study of 29 patients who received neoadjuvant imatinib (median duration, 8.5 months) for borderline resectable and locally advanced GIST, the overall response rate was $79.3 \%: 62.5 \%$ of the patients initially deemed to have unresectable GIST underwent surgery and $90.5 \%$ of those originally scheduled for surgery had a less extensive one [16]. Following resection, $86.2 \%$ of patients received adjuvant imatinib and, after a mean follow-up of 10 months, locoregional recurrence and distant metastasis were found in one $(4 \%)$ and two $(8 \%)$ patients, respectively [16].

In another study, 19 patients with locally advanced GIST received neoadjuvant imatinib $400 \mathrm{mg}$ /day for $\geq 12$ weeks (up to 6 months in cases of suboptimal response). Treatment was stopped 5-7 days prior to surgery and resumed afterward for 2 years (or until response) [17]. Preoperative imatinib allowed $68.4 \%$ of the patients to achieve PR with R0 resection, whereas $31.6 \%$ achieved SD with R1 resection. At a 2-year follow-up, there was no PD or death, and AEs were minimal [17]. Similarly, Jakob et al. followed up 36 patients with primary rectal GIST who underwent surgery in the context of neoadjuvant and/or adjuvant imatinib [18]. Results showed that the patients treated with perioperative imatinib had improved disease-free survival (DFS; $p<0.01)$ and OS $(p=0.03)$. Importantly, patients who received perioperative imatinib also had a higher rate of $\mathrm{R} 0$ resection compared with those who received adjuvant imatinib only $(p=0.02)$ [18].

In a longer-term analysis, 53 patients with primary GIST $\geq 5 \mathrm{~cm}$ (group A, $N=31$ ) or resectable metastatic/recurrent GIST $\geq 2 \mathrm{~cm}$ (group $\mathrm{B}, N=22$ ) received neoadjuvant imatinib $600 \mathrm{mg}$ /day for approximately 2 months and adjuvant therapy for 2 years [19]. Median TTP was not reached in group A and was 4.4 years in group B; 5-year DFS and OS rates were 57 and $77 \%$ in group A versus 30 and $68 \%$ in group B, respectively [19]. Moreover, imatinib was well tolerated.

Overall, these results demonstrate that perioperative imatinib improves resectability and survival outcomes for patients. The results also suggest that prior exposure to neoadjuvant imatinib does not compromise its efficacy as postsurgery adjuvant treatment to prevent recurrence. 
Table 2 Reintroduction of imatinib can be beneficial for patients who experience GIST recurrence following completion of prescribed adjuvant therapy

\begin{tabular}{|c|c|c|c|c|c|c|}
\hline Trial/study & Treatment (years) & $N$ & Follow-up (months) & Recurrence (\%) & TTP (months) & Response to reintroduction \\
\hline ACOSOG Z9001 [11] & $\begin{array}{l}\text { Placebo (1) } \\
\text { Imatinib (1) }\end{array}$ & $\begin{array}{l}354 \\
359\end{array}$ & 19.7 & $\begin{array}{l}20 \\
8\end{array}$ & $\begin{array}{l}\text { NA } \\
\sim 6\end{array}$ & NA \\
\hline SSGXVIII/AIO $[12,13]$ & $\begin{array}{l}\text { Imatinib (1) } \\
\text { Imatinib (3) }\end{array}$ & $\begin{array}{l}199 \\
198\end{array}$ & 54 & $\begin{array}{l}27.1 \\
13.6\end{array}$ & $\begin{array}{l}35.7 \\
35.7\end{array}$ & $84.8 \%$ of patients \\
\hline Kang [15] & Imatinib (2) & 1 & Ongoing & Yes & 10 & $43 \%$ smaller tumor 1 month later ${ }^{\mathrm{a}}$ \\
\hline
\end{tabular}

NA not applicable, TTP time to progression following imatinib cessation

${ }^{a}$ The patient experienced recurrence 3 months following reintroduction of imatinib $400 \mathrm{mg} /$ day and was being monitored after dose escalation to $800 \mathrm{mg} /$ day at the time of publication

Reintroduction of Imatinib after Treatment Interruption and Development of Drug Resistance

Resistance can be primary or secondary (Box 1). Primary resistance is defined by an early lack of response (typically within 3 to 6 months) and disease progression. It is often associated with mutations in KIT exon 9 or PDGFRA exon 18 (e.g., D842V) but is also found in wild-type GIST (i.e., GIST without known mutations in KIT or PDGFRA) [1, 20]. In contrast, secondary resistance generally occurs 6 to 24 months after patients' initial response to the treatment and is rarely seen in patients with PDGFRA mutations [20]. By definition, only secondary resistance is potentially amenable to imatinib reintroduction.

Clonal selection after acquisition of new KIT mutations, gene amplification, increased drug transport, and mutations in genes other than KIT and PDGFRA have been described to explain secondary resistance $[1,20]$. However, results from CML studies suggest that imatinib interruption does not promote survival of drug-resistant clones and development of secondary resistance [9]. Whether this is the case in GIST has not been extensively studied, but there is evidence

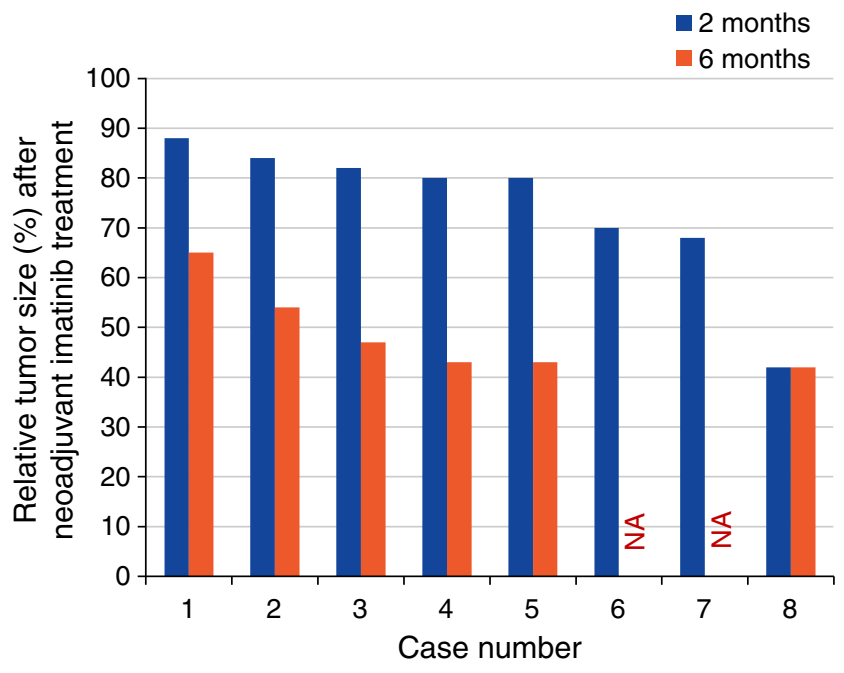

Fig. 1 Effect of neoadjuvant imatinib on tumor size. $N A$ not available [34] suggesting that the recurrence or progression during/after imatinib therapy does not necessarily equate with complete resistance to imatinib.

Lee et al. [21] studied 58 Korean patients with advanced GIST who had achieved at least SD while taking imatinib. After a median treatment time of 11.9 months, $24.1 \%$ of patients interrupted imatinib; 17.9 months later (median follow-up), $64.3 \%$ of patients who interrupted imatinib had developed PD, with a median PFS of 10.0 months. Median PFS from the time of imatinib initiation was 21.8 months (95\% CI, 17.3-26.3) in the interruption group but was not reached in the continuation group $(p=0.029)$. Importantly, $88 \%$ of the patients achieved disease control again following imatinib reintroduction, suggesting that in patients with nonprogressing GIST, it is possible to temporarily interrupt treatment when clinically warranted [21].

Another study by Blay et al. [10] indicated that $92 \%$ of patients who interrupted and then restarted imatinib treatment after experiencing GIST progression experienced tumor response or SD (Fig. 2). However, this particular study was not powered to support this endpoint, and an increased risk of resistance after reintroduction could not be excluded. In the prospective, multicenter, phase III BFR14 study, patients with advanced, nonprogressing GIST treated with imatinib were

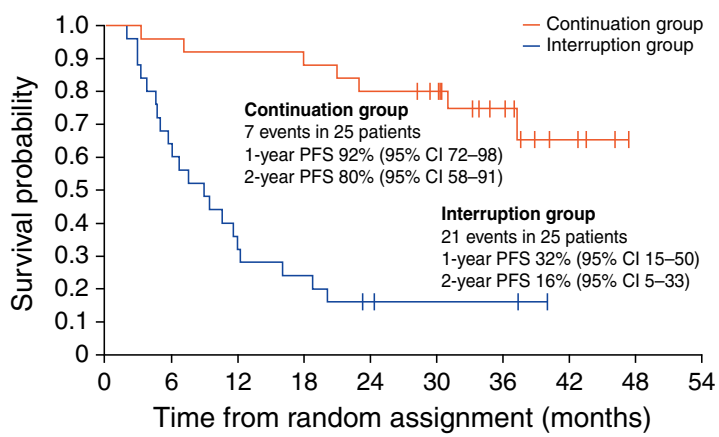

Number at risk Continuation group 25 Interruption group 25

$\begin{array}{ccc}23 & 20 & 10 \\ 8 & 3 & 2\end{array}$

Fig. 2 PFS in patients with advanced, nonprogressing GIST randomized to interruption or continuation of imatinib treatment at 3 years. (Reprinted from Blay et al. by the permission of Oxford University Press [7]) 
randomized to the interruption or continuation arm after 1 year ( $N=32$ versus 28$), 3$ years ( $N=25$ versus 25$)$, or 5 years ( $N=14$ versus 13$)$ of imatinib treatment $(400 \mathrm{mg} /$ day $)$, respectively $[22,23]$. After 18 months, $76 \%(54 / 71)$ of patients in the interruption arm restarted imatinib, $94.4 \%$ of whom had PD [23]. Imatinib reintroduction allowed tumor control in $94 \%$ of those patients, regardless of the length of prior imatinib therapy $[22,23]$. However, only 41 and $56 \%$ of patients who initially experienced $\mathrm{CR}$ and PR achieved a new $\mathrm{CR}$ and $\mathrm{PR}$ as best response, respectively, when imatinib was reintroduced (Table 3) [23]. Imatinib interruption had no effect on OS, but median PFS was shorter in the interruption arm than the continuation arm (Table 1): 7 versus 29 months (1 year, $p \leq 0.0001$ ), 9 months versus not reached ( 3 years, $p<0.0001$ ), and 13 months versus not reached (5 years), respectively [7,22]. These results suggest that the emergence of secondary resistance to imatinib decreases over time [7]. In addition, imatinib interruption had no effect on the time to secondary resistance, suggesting that it does not affect clonal selection of tumor cells in GIST. However, these results should be interpreted with caution because of the small number of patients in the study [7].

Overall, results from the BFR14 trial showed that interruption of imatinib therapy in patients with advanced disease correlates with a high risk of GIST progression and that reintroduction of imatinib remains an option for these patients. Imatinib interruption did not promote survival of drug-resistant clones or development of secondary resistance (Box 1), and reintroduction of imatinib could restore tumor control in most patients although at a lower level of efficacy than the initial therapy [7, 22, 23]. From a broader perspective, these results suggest that because imatinib is a TKI, it does not necessarily eradicate GIST tumor cells, resulting in persistent disease and the need for continuous suppression of the kinase activity of KIT or PDGFRA to achieve the best clinical outcomes [5]. Accordingly, these results emphasize the need to educate patients about the risk of interrupting imatinib therapy.

Table 3 Reintroduction of imatinib restores control of advanced GIST, but with a lower efficacy than the initial treatment [23]

\begin{tabular}{llll}
\hline $\begin{array}{l}\text { Randomized to imatinib interruption } \\
(N=51)\end{array}$ & $\begin{array}{l}\text { CR rate } \\
(\%)\end{array}$ & $\begin{array}{l}\text { PR rate } \\
(\%)\end{array}$ & $\begin{array}{l}\text { SD rate } \\
(\%)\end{array}$ \\
\hline $\begin{array}{l}\text { Best response prior to imatinib } \\
\text { interruption }\end{array}$ & 33.3 & 49 & 17.6 \\
$\begin{array}{l}\text { Best response following imatinib } \\
\text { reintroduction } \\
\quad \text { (relative to responding patients) }\end{array}$ & 41.2 & 56 & $\mathrm{NR}$ \\
$\begin{array}{l}\text { Best response following imatinib } \\
\text { reintroduction } \\
\text { (relative to total patients) }\end{array}$ & 13.7 & 27.5 & $\mathrm{NR}$ \\
\hline
\end{tabular}

$C R$ complete response, $N R$ not reported, $P R$ partial response, $S D$ stable disease
Dose escalation of Imatinib after Failure of Initial Treatment

A few studies have shown that dose escalation to 600 or $800 \mathrm{mg} /$ day may improve survival after patients experience $\mathrm{PD}$ on $400 \mathrm{mg} /$ day $[4,24,25]$, consistent with the idea that progression does not necessarily imply complete resistance to therapy. In a study of 47 evaluable patients with advanced GIST who experienced disease progression while treated with imatinib $400 \mathrm{mg} /$ day, $\mathrm{Li}$ et al. [24] reported that $40.4 \%$ achieved disease control when treated with imatinib $600 \mathrm{mg} /$ $\mathrm{ml}$ : $6.4 \%$ (3/47) had PR and $34.0 \%$ (16/47) had SD [24]. Median PFS for all patients was 17 weeks (95\% CI, 3.930.1 ), dose escalation to $600 \mathrm{mg} /$ day was well tolerated, and the most frequent AEs were edema and fatigue, reported by $73.1 \%$ of the patients. However, further dose escalation to $800 \mathrm{mg} /$ day in patients whose GIST progressed while on imatinib $600 \mathrm{mg} /$ day (14/47 or $29.7 \%$ ) was not effective [24].

In the phase III European Organization for Research and Treatment of Cancer/Italian Sarcoma Group/Australasian Gastrointestinal Trials Group (EORTC-ISG-AGITG) trial, 247 patients with advanced GIST who received imatinib $400 \mathrm{mg} /$ day were allowed to receive $800 \mathrm{mg}$ /day upon disease progression. Of the 241 patients available for follow-up, $55 \%$ (133) crossed over to the high-dose arm after exhibiting PD [25]. At the time of analysis, $2.3 \%$ of patients had PR, $27.1 \%$ had SD, and median PFS after crossover was 81 days. Overall, imatinib-related toxicities (mostly anemia and fatigue) remained easily manageable, and dose reductions after crossing over to the high-dose arm were necessary in $<8 \%$ of the cases [25]. In the phase III S0033 trial, 746 patients with advanced GIST were randomized to receive $400 \mathrm{mg}$ of imatinib once or twice daily, and those who progressed on $400 \mathrm{mg} / \mathrm{day}$ were allowed to cross over to the high-dose arm [26]. Of all patients whose dose was escalated to $400 \mathrm{mg}$ twice daily, $33 \%$ achieved PR or SD. Imatinib was relatively well tolerated, although serious AEs were more frequent in the high-dose arm (grade 3-5, $63 \%$ ) than the low-dose arm (grade $3-5,43 \%)$ [26].

Altogether, these results indicate that dose escalation is a feasible and reasonable option in patients with advanced, progressing GIST, despite evidence of increased toxicity. Furthermore, these results support the idea that GIST progression does not automatically mean resistance to imatinib.

\section{Reintroduction of Imatinib in Third- or Fourth-line Settings}

Although up to $80 \%$ of the patients with GIST will respond to imatinib treatment, 10-20\% will exhibit disease progression or recurrence associated with primary (early) resistance within 3-6 months of therapy initiation [20, 27]. In addition, 40 $50 \%$ of the patients will develop secondary (delayed or acquired) resistance and disease progression or recurrence after 6-24 months of therapy [27]. Several studies suggest 
that reintroduction of imatinib may be effective in GIST patients who develop resistance to first-line imatinib.

In a retrospective analysis, 223 patients with imatinib- and sunitinib-resistant metastatic GIST were treated with third-line nilotinib $(N=67)$, sorafenib $(N=55)$, or other drugs $(N=16)$. Alternatively, patients received imatinib plus another agent $(N=27)$, BSC $(N=18)$, or were re-treated with imatinib alone $(N=40)$ [28]. After adjustment for prognostic factors, results showed that nilotinib and sorafenib provided the best median PFS (4.1 versus 4.9 months, respectively) and median OS (11.8 versus 10.7 months, respectively) compared with BSC (PFS, 2.1 months, $p=0.001$; OS, 2.4 months, $p<0.0001$ ). Re-treatment with imatinib alone was also associated with improved OS (median, 7.5 months) compared with BSC $(p<0.0001)$ [28]. Similarly, reintroduction of imatinib in combination with other agents (doxorubicin, gemcitabine, cyclophosphamide, sirolimus, or bevacizumab) also improved OS (median, 8.7 months) compared with BSC $(p<0.0001)$, but how it compares to imatinib alone was not reported [28]. Reintroduction of imatinib thus represents a feasible option for patients with GIST that initially responded before developing secondary resistance (Box 1) and progressing on second-line therapies or becoming intolerant to other targeted therapies [29].

As mentioned previously, the most likely explanation for the response to imatinib reintroduction is that re-expansion of sensitive tumor clones occurs after the selection pressure is removed [29]. Results from one preclinical study suggest that autophagy, an evolutionarily conserved, lysosomal-driven process of controlled self-digestion, may contribute to the survival of GIST cells during imatinib-induced quiescence [30]. Gupta et al. indeed showed that autophagy is induced in GIST cell lines treated with imatinib in vitro and that inhibiting autophagy promotes cell death. Treatment of GIST-T1 and GIST882 cells with imatinib caused a specific three- to fivefold decrease in the $S+\mathrm{G}_{2} / \mathrm{M}$ fraction, with a matching increase in the $\mathrm{G}_{0} / \mathrm{G}_{1}$ fraction, indicating cell cycle arrest. In contrast, imatinib-resistant GIST-T1R cells did not undergo cell cycle arrest upon treatment [30]. Immunofluorescence and electron microscopy also showed formation of autophagosomes in imatinib-treated GIST-T1 cells, an effect that was reversed by RNA interference-mediated depletion of known autophagy regulators (ATG7 and ATG12). Importantly, analysis of tissue samples from patients treated with imatinib for 3, 5, or 7 days before resection revealed autophagosomes in responsive patients who received imatinib for 7 days compared with the shorter treatments [30]. Overall, results from preclinical experiments thus suggest that GIST cells could survive imatinib treatment by becoming quiescent and activating an autophagydependent survival mechanism.

Another preclinical study suggested instead that the presence of $\mathrm{KIT}^{\text {low }} \mathrm{CD} 44^{+} \mathrm{CD} 34^{+}$progenitor cells (or stem cells) that do not depend on KIT for survival explains the presence of residual, persistent, imatinib-refractory cells, and, consequently, the eventual recurrence/progression of GIST [31]. Regardless

\begin{tabular}{|c|}
\hline Patients 18 years and older with adva \\
\hline $\begin{array}{l}\text { Inclusion criteria: } \\
\text { - Prior benefit from imatinib for } \geq 6 \text { months } \\
\text { - Disease progressed despite prior imatinib ( } 400 \mathrm{mg} / \text { day) } \\
\text { and sunitinib treatment } \\
\text { - ECOG performance status } 0 \text { to } 3 \\
\text { - Adequate bone marrow function } \\
\text { - Adequate renal and hepatic function } \\
\text { - Life expectancy }>12 \text { weeks in the absence of } \\
\text { any intervention } \\
\text { - No other malignant disease apart from non-melanocytic } \\
\text { skin cancer or carcinoma in situ of the cervix or any } \\
\text { other cancer, except when treated with curative intent } \\
>5 \text { years previously without evidence of relapse } \\
\text { - Written, informed consent }\end{array}$ \\
\hline
\end{tabular}

Imatinib arm:

- Patients will receive imatinib 400 mg/day $(4 \times 100-\mathrm{mg}$ tablets) once daily with food until disease progression, unacceptable toxicity, or withdrawal of consent

\section{Exclusion criteria:}

- Medical and/or psychiatric conditions that compromise the patients' ability to give informed consent or complete protocol

- History of noncompliance

- Last dose of radiotherapy received within 4 weeks of the study start, except palliative radiotherapy

- Gl obstruction or active Gl bleeding

- Myocardial infarction within 6 months of the study start, or uncontrolled hypertension, congestive heart failure, or unstable angina

- Evidence of severe or uncontrolled systemic disease

- Female patients who are pregnant or breast-feeding (negative test within 1 week of starting imatinib required)

Placebo arm:

- Patients will receive placebo $400 \mathrm{mg} /$ day $(4 \times 100-\mathrm{mg}$ tablets) once daily with food until disease progression or withdrawal of consent

Endpoints:

- Primary endpoint: progression-free survival (time-frame: $\leq 12$ weeks)

- Secondary endpoint: disease control rate, i.e., complete or partial response and stable disease (time-frame: $\leq 12$ weeks)

Fig. 3 Diagram of the double-blind, randomized, phase III RIGHT trial to compare clinical outcomes following reintroduction of imatinib or placebo in patients with advanced/incurable GIST who benefited from prior imatinib but progressed on both imatinib and sunitinib 
of the mechanistic rationale for imatinib reintroduction, the NCCN recommends considering reintroduction of a previously tolerated and effective TKI for palliation of symptoms in patients with progressing GIST, despite prior response to imatinib and sunitinib [1]. According to NCCN guidelines, therapy should be continued for as long as patients can tolerate it as part of BSC.

Currently, a randomized, placebo-controlled, phase III trial (Rechallenge of Imatinib in GIST Having no effective Treatment [RIGHT]) is underway in Korea to compare outcomes following reintroduction of imatinib therapy versus placebo in patients with advanced/incurable GIST who initially responded but then progressed on imatinib and sunitinib [ClinicalTrials.gov identifier: NCT01151852] (Fig. 3). The study was expected to be completed in March 2013 and should provide additional insights on the effect of imatinib reintroduction on PFS and disease control rate. Several clinical studies are also completed or underway to investigate the efficacy of imatinib in combination with other drugs (e.g., everolimus [32], doxorubicin [33], and perifosine [ClinicalTrials.gov identifier: NCT00455559]) in treating metastatic GIST. Hopefully, these combinations will provide new options to re-treat tumors that previously responded to a single-agent TKI.

\section{Conclusions}

Current evidence indicates that imatinib potently inhibits the activity of KIT and PDGFRA kinases, but imatinib treatment may not completely eliminate GIST cells. Tumor control may thus depend on chronic, continuous suppression of imatinibsensitive tumor cells with imatinib to prevent GIST recurrence or progression [7, 15]. However, treatment holidays and discontinuations are not uncommon in advanced and adjuvant settings in GIST. These voluntary interruptions most often require patients to reinitiate therapy, and studies indicate that imatinib reintroduction can be effective. However, response to reintroduction may be suboptimal, and interruption of imatinib treatment should thus be avoided.

Overall, reintroduction of imatinib in patients with advanced/metastatic GIST can provide additional clinical benefits and improve patients' outcomes upon disease progression. Similarly, imatinib reintroduction offers clinical benefits in patients with GIST who completed adjuvant treatment and then experienced recurrence, independent of the length of prior adjuvant therapy. However, in both settings, reintroduction of imatinib after treatment interruption is associated with a lower efficacy and a higher rate of progressive disease, compared with initial therapy, suggesting that the tumor response to imatinib re-treatment may be suboptimal. Prior exposure to neoadjuvant imatinib did not appear to compromise the efficacy of postsurgical treatment either, which could be because the time elapsed between neoadjuvant and adjuvant treatments is typically short (few days).

Based on clinical evidence, treatment interruption does not appear to affect the emergence of imatinib-resistant clones, which likely explains why imatinib reintroduction can also provide clinical benefit as third- or fourth-line therapy in patients with GIST. Likewise, the apparent lack of effect of imatinib on emergence of resistance probably explains why dose escalation can be effective in controlling GIST growth in some patients.

In summary, the literature suggests that interruption of imatinib treatment in the adjuvant or metastatic setting neither compromises sensitivity to imatinib nor precludes its subsequent use to treat GIST recurrence or progression. Even though discontinuing or interrupting treatment is not recommended, due to increased risk of disease recurrence or progression, reintroduction of imatinib can lead to additional clinical benefits for patients with GIST and thus remains a feasible option for them in the adjuvant or advanced/ metastatic setting.

Acknowledgments Funding for medical writing and editorial assistance from Michele Jacob, PhD, and Larry Rosenberg, PhD, at Evidence Scientific Solutions was provided by Novartis Pharmaceuticals Corporation.

Conflict of Interest T. Reid is on the GIST speaker board for Gleevec (Novartis Pharmaceuticals).

\section{References}

1. National Comprehensive Cancer Network (NCCN) (2012.) NCCN Clinical Practice Guidelines in Oncology ${ }^{\mathrm{TM}}$ : Soft tissue sarcoma. National Comprehensive Cancer Network ${ }^{(\mathrm{R})}$. http://www.nccn.org/ professionals/physician_gls/f_guidelines.asp. Accessed September 21, 2012.

2. Blay JY, von Mehren M, Blackstein ME. Perspective on updated treatment guidelines for patients with gastrointestinal stromal tumors. Cancer. 2010;116(22):5126-37. doi:10.1002/cncr.25267.

3. Gleevec (imatinib mesylate) prescribing information, 2013, Novartis Pharma: East Hanover, NJ.

4. Blanke CD, Demetri GD, von Mehren M, Heinrich MC, Eisenberg $\mathrm{B}$, Fletcher JA, et al. Long-term results from a randomized phase II trial of standard- versus higher-dose imatinib mesylate for patients with unresectable or metastatic gastrointestinal stromal tumors expressing KIT. J Clin Oncol. 2008;26(4):620-5. doi:10.1200/JCO. 2007.13.4403.

5. Corless CL, Barnett CM, Heinrich MC. Gastrointestinal stromal tumours: origin and molecular oncology. Nat Rev Cancer. 2011;11(12):865-78. doi:10.1038/nrc3143.

6. Kee D, Zalcberg JR. Current and emerging strategies for the management of imatinib-refractory advanced gastrointestinal stromal tumors. Ther Adv Med Oncol. 2012;4(5):255-70. doi:10.1177/1758834012450935.

7. Blay JY, Pérol D, Le Cesne A. Imatinib rechallenge in patients with advanced gastrointestinal stromal tumors. Ann Oncol. 2012;23(7):1659 65. doi:10.1093/annonc/mdr622.

8. Agulnik M, Giel JL. Understanding rechallenge and resistance in the tyrosine kinase inhibitor era: Imatinib in gastrointestinal stromal tumor. Am J Clin Oncol. 2012. doi:10.1097/COC.0b013e31824be3d6. 
9. Mahon FX, Réa D, Guilhot J, Guilhot F, Huguet F, Nicolini F, et al. Discontinuation of imatinib in patients with chronic myeloid leukaemia who have maintained complete molecular remission for at least 2 years: the prospective, multicentre Stop Imatinib (STIM) trial. Lancet Oncol. 2010;11(11):1029-35. doi:10.1016/S1470-2045(10)70233-3.

10. Blay JY, Le Cesne A, Ray-Coquard I, Bui B, Duffaud F, Delbaldo C, et al. Prospective multicentric randomized phase III study of imatinib in patients with advanced gastrointestinal stromal tumors comparing interruption versus continuation of treatment beyond 1 year: the French Sarcoma Group. J Clin Oncol. 2007;25(9):1107-13. doi:10. 1200/JCO.2006.09.0183.

11. Dematteo RP, Ballman KV, Antonescu CR, Maki RG, Pisters PW, Demetri GD, et al. Adjuvant imatinib mesylate after resection of localised, primary gastrointestinal stromal tumour: a randomised, double-blind, placebo-controlled trial. Lancet. 2009;373(9669):1097104. doi:10.1016/S0140-6736(09)60500-6.

12. Reichardt P, Hartmann JT, Sundby Hall K, Eriksson M, Schütte J, Ramadori $\mathrm{G}$, et al. Response to imatinib rechallenge of GIST that recurs following completion of adjuvant imatinib treatment - the first analysis in the SSGXVIII/AIO trial patient population. Eur J Cancer. 2011;47 Suppl 2:31LBA. abstr.

13. Joensuu H, Eriksson M, Sundby Hall K, Hartmann JT, Pink D, Schütte $J$, et al. One vs three years of adjuvant imatinib for operable gastrointestinal stromal tumor: a randomized trial. JAMA. 2012;307(12):1265-72. doi:10.1001/jama.2012.347.

14. DeMatteo RP, Lewis JJ, Leung D, Mudan SS, Woodruff JM, Brennan MF. Two hundred gastrointestinal stromal tumors: recurrence patterns and prognostic factors for survival. Ann Surg. 2000;231(1):51-8.

15. Kang YK. Response to imatinib rechallenge in a patient with a recurrent gastrointestinal stromal tumor after adjuvant therapy: a case report. J Med Case Rep. 2011;5:504. doi:10.1186/1752-1947-5-504.

16. Shrikhande SV, Marda SS, Suradkar K, Arya S, Shetty GS, Bal M, et al. Gastrointestinal stromal tumors: case series of 29 patients defining the role of imatinib prior to surgery. World J Surg. 2012;36(4):864-71. doi:10.1007/s00268-012-1440-4.

17. Ashraf M, Jha J, Choudhry A, Aggarwal B, Nayak S, Chakraborty J, et al. Neoadjuvant and adjuvant therapy with imatinib for locally advanced gastrointestinal stromal tumors in eastern Indian patients. Asian Pac J Cancer Prev. 2011;12(8):2059-64.

18. Jakob J, Mussi C, Ronellenfitsch U, Wardelmann E, Negri T, Gronchi A, et al. Gastrointestinal stromal tumor of the rectum: results of surgical and multimodality therapy in the era of imatinib. Ann Surg Oncol. 2012;20(2):586-92. doi:10.1245/s10434-012-2647-1.

19. Wang D, Zhang Q, Blanke CD, Demetri GD, Heinrich MC, Watson $\mathrm{JC}$, et al. Phase II trial of neoadjuvant/adjuvant imatinib mesylate for advanced primary and metastatic/recurrent operable gastrointestinal stromal tumors: long-term follow-up results of Radiation Therapy Oncology Group 0132. Ann Surg Oncol. 2012;19(4):1074-80. doi:10.1245/s10434-011-2190-5.

20. Gounder MM, Maki RG. Molecular basis for primary and secondary tyrosine kinase inhibitor resistance in gastrointestinal stromal tumor. Cancer Chemother Pharmacol. 2011;67 Suppl 1:S25-43. doi:10. 1007/s00280-010-1526-3.

21. Lee JL, Ryu MH, Chang HM, Kim TW, Kang HJ, Sohn HJ, et al. Clinical outcome in gastrointestinal stromal tumor patients who interrupted imatinib after achieving stable disease or better response. Jpn J Clin Oncol. 2006;36(11):704-11. doi:10.1093/jjco/hyl088.

22. Le Cesne A, Ray-Coquard IL, Bui Nguyen B, Adenis A, Rios M, Bertucci F, et al. Time to secondary resistance (TSR) after interruption of imatinib (IM) in advanced GIST: updated results of the prospective French Sarcoma Group randomized phase III trial on long-term survival. J Clin Oncol. 2011;29:10015. (Suppl): abstr.

23. Domont J, Blay J, Ray-Coquard IL, Bui Nguyen B, Adenis A, Rios M, Bertucci F, Duffaud F, Cupissol D, Chevreau C, Bompas E, Bourne-Branchu V, Chabaud S, Le Cesne A (2011) Influence of imatinib interruption and imatinib rechallenge on the residual tumor volume in patients with advanced GIST: Results of the BFR14 prospective French Sarcoma Group randomized phase III trial. J Clin Oncol 29(Suppl):abstr 10054.

24. Li J, Gong JF, Gao J, Sun NP, Shen L. Efficacy of imatinib dose escalation in Chinese gastrointestinal stromal tumor patients. World $\mathrm{J}$ Gastroenterol. 2012;18(7):698-703. doi:10.3748/wjg.v18.i7.698.

25. Zalcberg JR, Verweij J, Casali PG, Le Cesne A, Reichardt P, Blay JY, et al. Outcome of patients with advanced gastro-intestinal stromal tumours crossing over to a daily imatinib dose of $800 \mathrm{mg}$ after progression on $400 \mathrm{mg}$. Eur J Cancer. 2005;41(12):1751-7. doi:10. 1016/j.ejca.2005.04.034.

26. Blanke CD, Rankin C, Demetri GD, Ryan CW, von Mehren M, Benjamin RS, et al. Phase III randomized, intergroup trial assessing imatinib mesylate at two dose levels in patients with unresectable or metastatic gastrointestinal stromal tumors expressing the kit receptor tyrosine kinase: S0033. J Clin Oncol. 2008;26(4):626-32. doi:10. 1200/JCO.2007.13.4452.

27. Zalcberg JR, Desai J. Dose optimization of tyrosine kinase inhibitors to improve outcomes in GIST. Asia Pac J Clin Oncol. 2012;8(1):4352. doi:10.1111/j.1743-7563.2011.01491.x.

28. Italiano A, Cioffi A, Coco P, Maki RG, Schöffski P, Rutkowski P, et al. Patterns of care, prognosis, and survival in patients with metastatic gastrointestinal stromal tumors (GIST) refractory to first-line imatinib and second-line sunitinib. Ann Surg Oncol. 2012;19(5):1551-9. doi:10.1245/s10434-011-2120-6.

29. Fumagalli E, Coco P, Morosi C, Bertulli R., Casali P (2010) Sunitinib rechallenge in two advanced GIST patients after third-line antityrosine kinase therapy. J Clin Oncol 28(Suppl):abstr e20519.

30. Gupta A, Roy S, Lazar AJ, Wang WL, McAuliffe JC, Reynoso D, et al. Autophagy inhibition and antimalarials promote cell death in gastrointestinal stromal tumor (GIST). Proc Natl Acad Sci U S A. 2010;107(32):14333-8. doi:10.1073/pnas.1000248107.

31. Bardsley MR, Horvath VJ, Asuzu DT, Lorincz A, Redelman D, Hayashi Y, et al. Kitlow stem cells cause resistance to Kit/plateletderived growth factor alpha inhibitors in murine gastrointestinal stromal tumors. Gastroenterology. 2010;139(3):942-52. doi:10. 1053/j.gastro.2010.05.083.

32. Schöffski P, Reichardt P, Blay JY, Dumez H, Morgan JA, RayCoquard I, et al. A phase I-II study of everolimus (RAD001) in combination with imatinib in patients with imatinib-resistant gastrointestinal stromal tumors. Ann Oncol. 2010;21(10):1990-8. doi:10. 1093/annonc/mdq076.

33. Maurel J, Martins AS, Poveda A, López-Guerrero JA, Cubedo R, Casado A, et al. Imatinib plus low-dose doxorubicin in patients with advanced gastrointestinal stromal tumors refractory to high-dose imatinib: a phase I-II study by the Spanish Group for Research on Sarcomas. Cancer. 2010;116(15):3692-701. doi:10.1002/cncr.25111.

34. Haller F, Detken S, Schulten HJ, Happel N, Gunawan B, Kuhlgatz J, et al. Surgical management after neoadjuvant imatinib therapy in gastrointestinal stromal tumours (GISTs) with respect to imatinib resistance caused by secondary KIT mutations. Ann Surg Oncol. 2007;14(2):526-32. doi:10.1245/s10434-006-9228-0. 